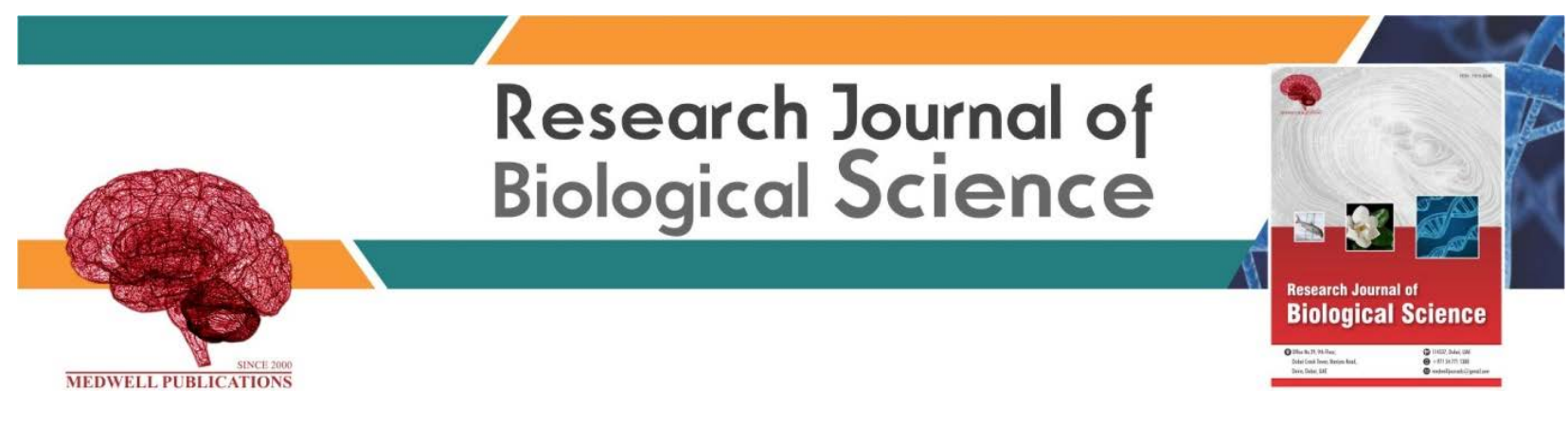

\title{
Effect of Chronic Renal Dialysis on the Level of Thyroid Gland Hormones among Patients with Chronic Renal Failure
}

\author{
${ }^{1}$ Kamal Mansi, ${ }^{1}$ Saif Altamemi, ${ }^{2}$ Sami Aloush and ${ }^{2}$ Mohammed Albashtawy \\ ${ }^{1}$ Department of Medical Labaratory Sciences, Faculty of Sciences, Mafraq, Jordan \\ ${ }^{2}$ Faculty of Nursing, Al-Bayt University, Mafraq, Jordan
}

Key words: CRF, haemodialysis, thyroid hormones, creatinine, urea, haemodialysis

\author{
Corresponding Author: \\ Kamal Mansi \\ Sciences, Mafraq, Jordan \\ Page No.: 1-4 \\ Volume: 15, Issue 1, 2020 \\ ISSN: $1815-8846$ \\ Research Journal of Biological Sciences \\ Copy Right: Medwell Publications
}

Department of Medical Labaratory Sciences, Faculty of
Abstract: The purpose of this study was to assess the effect of haemodialysis on thyroxin level in patients with chronic renal failure. The sample of this study included forty nine participants (28 male and 21 female). Eligible participants were patients on regular dialysis for $>1$ year at the time of enrollment, had no co morbidity and had no known previous thyroid dysfunction. The study was conducted in one educational hospital in Jordan. Serum level of thyroid hormones T4 and T3 were measured prior to the dialysis session and after completion. There was a statistically significant increase in the level of T3 and T4 after completion of dialysis, however, this increase did not go beyond the normal value. Haemodialysis initiate a trend for increase in the level of thyroxin hormones. Patients on chronic dialysis need vigilant assessment for thyroid function in order to prevent future complications.

\section{INTRODUCTION}

Chronic Renal Failure (CRF) is defined as kidney impairment that can last for $>3$ months and is associated with decrease urine production and significant build up of waste products ${ }^{[1]}$. Although, chronic renal failure is a common complication for endocrine disorders ${ }^{[2,3]}$ many of these disorders are asymptomatic which made diagnosis of these disturbances hard. Laboratory values have been the mainstay in the diagnosis of thyroid dysfunction $^{[4,5]}$.

Several conditions are associated with thyroid hormone deficiency such as hypopituitarism and impairment of synthesis within the thyroid gland itself. Thyroid activity is regulated by the pituitary gland which secrete the Thyroid Stimulating Hormone $(\mathrm{TSH})^{[6,7]}$. Hypothyroidism can be classified into three types; primary, secondary and cellular ${ }^{[8,9]}$. Primary hypothyroidism is diagnosed when there is an abnormal elevation in the level of TSH coupled with a significant decrease in the level of thyroid hormone. Secondary hypothyroidism is caused by pituitary gland malfunction and is diagnosed when there is significant decrease in the level of Thyroid Stimulating Hormone (TSH), Triiodothyronine (T3) and Thyroxine (T4). Thyroid gland cellular dysfunction is the main pathology that causes cellular hypothyroidism and is usually associated with low basal metabolic rate. It is diagnosed when there is normal serum level of $\mathrm{T} 3, \mathrm{~T} 4^{[10]}$.

Studies reported significant increase in the risk of goiter and other thyroid related insufficiency among patients with end stage renal diseases in comparison with their counterparts of normal health status ${ }^{[11,12]}$.

Haemodialysis serves to remove waste products and extra fluid from blood, however, it has been postulated that long term dialysis therapy may affect thyroid functioning ${ }^{[13,14]}$. Few studies have investigated the effect of dialysis on thyroid function among patients on long 
term dialysis and thus, this study was conducted to assess the effect of dialysis on the level of thyroid hormones among dialysis patients.

\section{MATERIALS AND METHODS}

Design: This study used a quasi-experimental design with one group pre-post test comparison.

Participants: Eligible participants where patients who had diagnostic symptoms of chronic renal failure had no past health history of thyroid dysfunction, underwent dialysis for at least 1 year and had no co-morbidity.

Sample: $G$ power software ${ }^{[15]}$ was used to calculate the sample size for this study. Based on power of 0.9, level of significance at 0.05 and an estimated medium effect size $\mathrm{d}=0.5$ the required sample size to run two tailed paired sample t-test was 44 . This study collected a convenience sample of 49 participants to compensate for the expected drop out rate.

Setting: This study was conducted in the renal dialysis unit of one governmental hospital in the North of Jordan. The unit capacity was XXX beds. The unit performs XXX dialysis sessions a day. The hospital was selected based on the reasonably large capacity, the availability of dialysis unit and the ethical permission to conduct the study. Patients in this unit usually undergo 3 dialysis sessions per week with 4 sessions per hour.

Ethical permission: This study was granted ethical permission from the institutional review board at the principal investigator's university and the participating hospital.

Data collection: At the day of the study, participants were approached in the dialysis unit, explanation about the study was provided and written informed consent was obtained. A data collection form was made for this study that included items about age, sex and duration of dialysis, family history and presence of co-morbidity. Venous blood sample was obtained. Two blood samples were obtained from every patient hematology and chemistry. A master code list with the participants names and codes was created. Data were entered and stored on the principal investigator with restricted access. All hardcopies materials related to the study were appropriately discarded.

Sample collection: The blood sample was collected from patients in the morning immediately before and after hemodialysis. The haematolgy blood sample were collected using EDTA bottles tubes and used for determining the hematological parameters described below. The chemistry blood sample were allowed to settle for $30 \mathrm{~min}$ and then centrifuged using a bench top centrifuge to collect plasma/serum.

Hematological analysis: Complete Blood Count (CBC) was performed using the automated hematology analyzer.

Biochemical analysis: Urea and creatinine quantization was performed using commercial analytical kits from Sigma (Lab-Kit, Spain).

Thyroid function assay: Total $\mathrm{T} 3$ and $\mathrm{T} 4$ were determined by using the total Triiodothyronine $\mathrm{T} 3$ and the total thyroxin T4 immunoassay test kits (Biocheck, INC, USA). TSH concentration was measured using the Enzyme assay for the ultrasensitive Quantitative determination of TSH in human serum (Biocheck, INC, USA).

Statistical analyses: Means and standard deviations were calculated and two-sample t-test and Mann-Whitney Utest was used to compare the change of hormones before and after dialysis.

\section{RESULTS AND DISCUSSION}

The studied sample included 28 males forming $57.1 \%$ of the sample of the participants only $8.2 \%$ of the samples were $<20$ years of age while $28.6 \%$ were $21-40$ years of age. The highest percentage of sample age ranged from 41-60 years with $44.9 \%$. Participants who were older than were $18.4 \%$ (Table 1). Bulk of sample (42.9\%) has renal failure since, $<5$ years while $26.5 \%$ since, $5-10$ years, $16.3 \%$ since, $11-15$ years and $4.1 \%$ had renal failure for 16 years or more.

Our data showed statistically significant increase in the levels of T3 and T4 following dialysis; however, the change in the level of TSH was not significant (Table 1). In addition, female participants showed much better increase in $\mathrm{T} 3$ and $\mathrm{T} 4$ when compared with male (Table 2).

Our findings were consistent with other studies that revealed significant effect for dialysis on the levels of T3, $\mathrm{T} 4{ }^{[16]}$. One study reported that dialysis stimulated a trend for hypothyroidism in patients with chronic renal failure ${ }^{[17]}$. One reasons for this finding were the reduction in the concentration of thyroxin binding globulin and the presence of thyroid hormone binding inhibitors ${ }^{[18]}$.

Other studies in support to this study have shown that increase in serum levels of toxic compounds in dialysis patients. That entailed diminishing serum levels of thyroid hormones $^{[19]}$ another reason is that iodide excretion is diminished in chronic renal failure, leading sequentially to an elevated plasma inorganic iodide concentration and an initial increment in thyroidal iodide uptake ${ }^{[20]}$. Increases in total body inorganic iodide can potentially block thyroid hormone production. 
Res. J. Biol. Sci., 15 (1): 1-4, 2020

Table 1: The effect of dialysis on thyroid function (T4, T3, TSH, urea and creatinine)

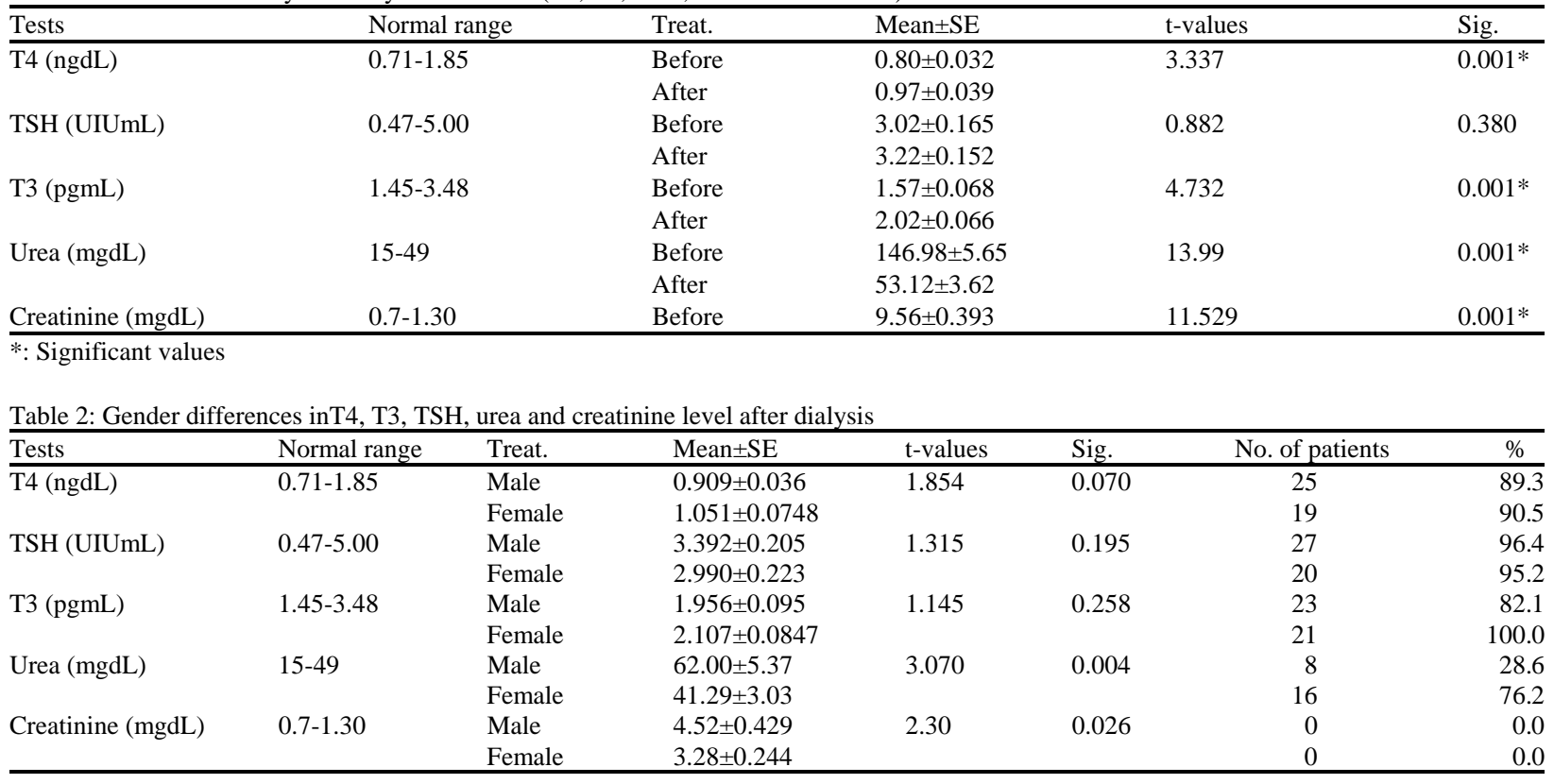

\section{CONCLUSION}

Patients who undergo dialysis are at high risk for thyroid gland disturbances. Therefore, it is important that patients who show increased levels of T3 and T4 be carefully assessed and treated in order to prevent significant deterioration in thyroid gland function.

\section{ACKNOWLEDGEMENT}

We thank the Al-Bayt University, Al-Mafraq, Jordan for the financial support. Researechers also would like to thank the nursing staff in Princess Bassam Hospital in Irbid for their valuable assistant and support. We also, thank all patients who participated in this study.

\section{REFERENCES}

01. Razmaria, A.A., 2016. Chronic kidney disease. Jama Patient Page, 315: 2248-2248.

02. Kuczera, P., M. Adamczak and A. Wiecek, 2015. Endocrine abnormalities in patients with chronic kidney disease. Prilozi, 36: 109-118.

03. Singh, A.K., A. Raed and J. Kari, 2015. Endocrine Complications of Chronic Kidney Disease Chronic Renal Disease. In: Chronic Kidney Diseases, Kimmel, P.L. and M.E. Rosenberg (Eds.). Elsevier, New York, USA., pp: 310-319.

04. Mohamedali, M., S. Reddy Maddika, A. Vyas, V. Iyer and P. Cheriyath, 2014. Thyroid disorders and chronic kidney disease. Intl. J. Nephrol., 2014: 1-6.
05. Parsa, A.A. and H. Gharib, 2018. Laboratory Evaluation for Thyroid Nodules. In: Thyroid Nodules: Contemporary Endocrinology, Gharib, H. (Ed.). Humana Press, Cham, Switzerland, ISBN: 978-3-319-59473-6, pp: 19-33.

06. Tziaferi, V. and M.T. Dattani, 2018. Pituitary Gland Embryology, Anatomy and Physiology. In: Endocrine Surgery in Children, Ledbetter, D. and P. Johnson (Eds.). Springer, Berlin, Germany, ISBN: 978-3-662-54254-5, pp: 427-437.

07. Zhang, Q., L. Zang, Y.J. Li, B.Y. Han and W.J. Gu et al., 2018. Thyrotrophic status in patients with pituitary stalk interruption syndrome. Med., 97: 1-4.

08. Gupta, V. and M. Lee, 2011. Central hypothyroidism. Indian J. Endocrinol. Metab., 15: S99-S106.

09. Kostoglou-Athanassiou, I. and K. Ntalles, 2010. Hypothyroidism-new aspects of an old disease. Hippokratia, 14: 82-87.

10. Persani, L., 2012. Central hypothyroidism: Pathogenic, diagnostic and therapeutic challenges. J. Clin. Endocrinol. Metab., 97: 3068-3078.

11. Lei, W.H., C.X. Shao, J. Xin, J. Li and M.F. Mao et al., 2016. Multinodular goiter spontaneous hemorrhage in ESRD patients result in acute respiratory failure: A case report. Med., 95: 1-4.

12. Lo, J.C., G.J. Beck, G.A. Kaysen, C.T. Chan and A.S. Kliger et al., 2017. Thyroid function in end stage renal disease and effects of frequent hemodialysis. Hemodialysis Intl., 21: 534-541. 
13. Malik, Y.O., S.M. Raza and S. Arunselvan, 2015. Coexisting tertiary hyperparathyroidism and severe hypothyroidism in an end-stage renal disease patient on hemodialysis. Nephrourol. Mon., 7: 1-3.

14. So, E. and R. Arakaki, 2014. Toxic multinodular goiter in a patient with end-stage renal disease and hemodialysis. Hawaii J. Med. Pub. Health, 73: 217-220.

15. Erdfelder, E., F. Faul and A. Buchner, 1996. GPOWER: A general power analysis program. Behav. Res. Methods Instrum. Comput., 28: 1-11.

16. Iglesias, P. and J.J. Dies, 2009. Thyroid dysfunction and kidney disease. Eur. J. Endocrinol., 160: 503-515.
17. Connor, A. and J.E. Taylor, 2008. Renal impairment resulting from hypothyroidism. NDT. Plus, 1: 440-441.

18. Pakhle, K., R. Parikh, A. Jain, P. Kashyap and D. Dave et al., 2017. Thyroid dysfunctions in patients with chronic renal failure. Intl. J. Res. Med. Sci., 5: 2773-2778.

19. Kaggia, S.N.N., 2013. Thyroid hormone profiles in patients with chronic kidney disease at kenyatta national hospital. MSc Thesis, University of Nairobi, Nairobi, Kenya.

20. Palmer, B.F. and W.L. Henrich, 2011. Thyroid function in chronic kidney disease. Kidney Intl., 24: 11-13. 